\title{
Clinical and Histological Predictors of Renal Survival in Patients with Biopsy-Proven Diabetic Nephropathy
}

\author{
Ting Zhou ${ }^{a}$ Yiyun Wang ${ }^{b}$ Li Shen ${ }^{c}$ Xiaomei Li ${ }^{a}$ Qiong Jiao ${ }^{a} \quad$ Ze Li $^{a}$ \\ Junjie Jia ${ }^{\mathrm{a}} \mathrm{Li} \mathrm{He}^{\mathrm{a}}$ Qunzi Zhang ${ }^{\mathrm{a}}$ Niansong Wang ${ }^{\mathrm{a}}$ Ying Fan ${ }^{\mathrm{a}}$ \\ aDepartment of Nephrology, Shanghai Jiao Tong University Affiliated Sixth People's Hospital, Shanghai, China; \\ bepartment of Emergency, Shanghai United Family Hospital, Shanghai, China; 'Clinical Research Unit, Shanghai \\ Jiao Tong University Affiliated Sixth People's Hospital, Shanghai, China
}

\author{
Keywords \\ Diabetic nephropathy · Clinical characteristic · Pathology · \\ Renal biopsy · Prediction
}

\begin{abstract}
Introduction: Clinical indicators or pathological features alone cannot reliably predict renal survival in patients with biopsy-confirmed diabetic nephropathy (DN). Therefore, this analysis sought to develop and validate a predictive model incorporating both clinical and pathological markers to predict renal outcomes in patients with biopsy-confirmed DN. Methods: A predictive nomogram was developed based upon data pertaining to 194 patients with biopsy-confirmed DN. The prognostic relevance of individual clinicopathological variables was assessed through univariate and multivariate Cox regression analyses. A prognostic nomogram was then developed and validated based upon concordance (C)index values and calibration curves. Internal validation was conducted through bootstrap resampling, while the clinical utility of this model was assessed via a decision curve analysis (DCA) approach. Results: Nephrotic-range 24-h proteinuria, late-stage CKD, glomerular classification III-IV, and IFTA score 2-3 were all identified as independent predictors of poor renal outcomes in DN patients and were incorporated
\end{abstract}

(C) 2021 The Author(s)

Published by S. Karger AG, Basel

This is an Open Access article licensed under the Creative Commons Attribution-NonCommercial-4.0 International License (CC BY-NC) (http://www.karger.com/Services/OpenAccessLicense), applicable to the online version of the article only. Usage and distribution for commercial purposes requires written permission. into our final nomogram. Calibration curves revealed good agreement between predicted and actual 3-and 5-year renal survival in DN patients with the $\mathrm{C}$-index value for this nomogram at 0.845 (95\% Cl: 0.826-0.864). DCA revealed that our nomogram was superior to models based solely upon clinical indicators. Conclusion: A predictive nomogram incorporating clinical and pathological indicators was developed and validated for the prediction of renal survival outcomes in patients with biopsy-confirmed DN. This model will be of value to clinicians, as it can serve as an easy-to-use and reliable tool for physicians to guide patient management based on individualized risk.

(c) 2021 The Author(s)

Published by S. Karger AG, Basel

\section{Introduction}

Diabetic nephropathy (DN) is a serious complication that can arise in patients suffering from diabetes mellitus (DM), and it is among the most common global drivers of end-stage renal disease (ESRD). As rates of DM continue to rise throughout the world, DN incidence will inevitably increase, imposing a significant social and finan-

Ting Zhou and Yiyun Wang contributed equally to the study.
Correspondence to:

Niansong Wang, wangniansong2012@163.com

Ying Fan, fanyingsh@126.com 
cial burden on affected individuals and nations [1-3]. It is thus vital that clinicians be able to predict renal outcomes for $\mathrm{DN}$ patients during the early stages of disease, so that they can provide individualized care to all patients.

No reliable predictors of renal survival are currently available for DN patients. While reduced eGFR and albuminuria are frequently used as biomarkers of abnormal renal function in patients with DN [4-6], multiple studies have highlighted inconsistencies between these indicators and actual renal impairment in this patient population $[7,8]$. For example, normoalbuminuric renal insufficiency has been shown to affect $22-24 \%$ and $32-71 \%$ of type 1 and type $2 \mathrm{DM}$ patients, respectively $[9,10]$. Although decreased eGFR is also often evaluated as a biomarker of renal injury, its relationship with clinical outcomes has only been clearly observed in patients with severe proteinuria, and its relevance in those with early-stage disease is unclear [11]. As such, reliance upon albuminuria and decreased eGFR has led to the underdiagnosis of many patients with histologically proven $\mathrm{DN}[12,13]$. These clinical indicators are thus insufficient for use in predicting renal survival in $\mathrm{DN}$ patients.

The relationship between particular pathological parameters, $\mathrm{DN}$ severity, and patient prognosis has also been evaluated following the pathological classification of $\mathrm{DN}$ by Tervaert et al. [14]. Glomerular lesions, arteriosclerosis, and interstitial fibrosis and tubular atrophy (IFTA) are all closely associated with long-term renal outcomes [15]. Even DN patients with normal albuminuria levels and an eGFR $<60$ $\mathrm{mL} / \mathrm{min} / 1.732$ may still exhibit serious tubulointerstitial injury without any differences in renal tubulointerstitial pathological changes when compared with those of DM patients with macroalbuminuria [11], with potential variability being attributable to sampling site, ethnicity, or other factors. As such, clinical and pathological factors alone are insufficient to accurately predict DN patient renal outcomes, potentially resulting in the delayed treatment of patients at a high risk of renal failure. Hence, we sought to design a more reliable predictive model incorporating clinical and pathological variables in order to more reliably stratify $\mathrm{DN}$ patients based upon their individual levels of renal risk in an effort to better guide patient treatment and to improve associated outcomes.

\section{Materials and Methods}

\section{Patients and Study Design}

This study consisted of a retrospective analysis of data from T2DM patients that underwent renal biopsy at the Shanghai Sixth People's hospital from January 2008 to April 2020. In total, 194 patients were eligible for this study. T2DM was diagnosed according to the American Diabetes Association (ADA) criteria [3], while the 2010 DN classification system was used for the diagnosis of DN [14]. Diabetic retinopathy (DR) was defined by the presence of microaneurysms, retinal hemorrhages, soft/hard exudates, or vitreous hemorrhage [16]. Patients were excluded from this study if they did not have T2DM, suffered from nondiabetic nephropathy, had a poorly defined medical history, incomplete clinical dataset, had an eGFR $<30 \mathrm{~mL} / \mathrm{min} / 1.73 \mathrm{~m}^{2}$, had $<5$ glomeruli in the renal biopsy sample, or suffered from comorbidities including tumors, serious infections, systemic lupus erythematosus, or Henoch Schonlein purpura nephritis. In addition, patients that had undergone renal transplant or dialysis prior to biopsy were also excluded (shown in online suppl. Fig. 1; for all online suppl. material, see www.karger.com/doi/10.1159/000518222).

The Ethics Committee of the Shanghai Jiao Tong University Affiliated Sixth People's Hospital approved this study, which was consistent with the Declaration of Helsinki. Informed consent was obtained from all patients.

\section{Clinical and Laboratory Data}

Clinical data collected for all patients included age, sex, DM disease course, insulin and oral hypoglycemic drug use, hypertension status, renin-angiotensin-aldosterone system inhibitor use, family history of DM, the presence of DR, systolic blood pressure, and diastolic blood pressure. Standard laboratory measures were used to quantify glycosylated hemoglobin A1c, serum creatinine ( $\mathrm{sCr}$ ), blood urea nitrogen, cystatin C, uric acid, eGFR (calculated via the MDRD formula), presence of glomerular hematuria, 24-h proteinuria, serum calcium, hemoglobin, serum albumin, total triglyceride, serum phosphorus, total cholesterol, high-density lipoprotein cholesterol, and low-density lipoprotein cholesterol levels.

\section{Renal Biopsy and Classification}

Renal biopsy was conducted for these 194 patients for the following indications: proteinuria $>3.5 \mathrm{~g}$ /day with normal renal function; a rapid decline in renal function with 3 months; the presence of glomerular hematuria; degree of proteinuria and renal functional damage that were not consistent with the course of DM, with a DM course $<5$ years; and the presence of renal functional damage without retinopathy. All biopsy samples were used to prepare $3-\mu \mathrm{m}$ sections that were used for periodic acid Schiff, Masson's trichrome, and hematoxylin and eosin staining. Two pathologists independently reviewed all samples. DN pathological classification was based upon the Research Committee of the Renal Pathology Society system proposed in 2010 [14].

\section{Follow-Up and Endpoints}

Renal endpoints analyzed in this study included a doubling of baseline sCr, first-onset ESRD, initiation of renal replacement therapy, or death. The median patient follow-up time was 30 months.

\section{Statistical Analysis}

SPSS 21.0 (SPSS Inc., Chicago, IL, USA) and R v.4.0.2 (http:// www.R-porject.org/) were used for all statistical testing. Categorical data were given as frequencies (percentages), while normally and nonnormally distributed continuous data were given as mean \pm SD and median with interquartile range, respectively. These 3 data types were compared via $\chi^{2}$ tests, Student's $t$ tests, and Mann- 
Table 1. Clinical characteristics of patients with diabetic nephropathy

\begin{tabular}{|c|c|c|c|c|}
\hline \multirow[t]{2}{*}{ Variables } & \multirow{2}{*}{$\begin{array}{l}\text { Total } \\
\text { patients }(n=194)\end{array}$} & \multicolumn{2}{|l|}{ Renal endpoint events } & \multirow[t]{2}{*}{$p$ value } \\
\hline & & yes $(n=81)$ & no $(n=113)$ & \\
\hline Age, years & $56(46-62)$ & $55(45-61)$ & $56(49-63)$ & 0.268 \\
\hline Sex, male, $n(\%)$ & $137(70.6)$ & $54(66.7)$ & $83(73.5)$ & 0.306 \\
\hline Course of DM, years & $10(6-15)$ & $10(6-16)$ & $10(5-15)$ & 0.195 \\
\hline Family history of DM, $n(\%)$ & $96(49.5)$ & $44(55.0)$ & $52(46.4)$ & 0.242 \\
\hline Use of OHA, $n(\%)$ & $160(82.5)$ & $70(86.4)$ & $90(79.6)$ & 0.221 \\
\hline Use of insulin, $n(\%)$ & $161(83.0)$ & $68(89.5)$ & $93(82.3)$ & 0.200 \\
\hline Use of RAAS inhibitor, $n(\%)$ & $154(79.4)$ & $63(77.8)$ & $91(80.5)$ & 0.312 \\
\hline $\mathrm{DR}, n(\%)$ & $118(60.8)$ & $62(86.1)$ & $56(58.3)$ & $<0.001$ \\
\hline $\mathrm{SBP}, \mathrm{mm} \mathrm{Hg}$ & $140 \pm 20$ & $142 \pm 20$ & $138 \pm 20$ & 0.120 \\
\hline DBP, mm Hg & $80(75-90)$ & $83(75-90)$ & $80(75-87)$ & 0.251 \\
\hline $\mathrm{HbA1c}, \%$ & $7.60(6.50-9.28)$ & $7.50(6.40-9.42)$ & $7.60(6.70-9.20)$ & 0.335 \\
\hline $\mathrm{sCr}, \mu \mathrm{mol} / \mathrm{L}$ & $106(79-132)$ & $129(105-170)$ & $90(69-115)$ & $<0.001$ \\
\hline Uric acid, $\mu \mathrm{oml} / \mathrm{L}$ & $371 \pm 89$ & $375 \pm 92$ & $368 \pm 87$ & 0.585 \\
\hline $\mathrm{BUN}, \mathrm{mmol} / \mathrm{L}$ & $7.40(5.89-9.70)$ & $8.8(7.2-11.1)$ & $6.6(5.5-8.4)$ & $<0.001$ \\
\hline Cystatin C, mg/L & $1.4(1.0-2.0)$ & $1.9(1.6-2.3)$ & $1.2(1.0-1.5)$ & $<0.001$ \\
\hline $\mathrm{eGFR}, \mathrm{mL} / \mathrm{min} / 1.73 \mathrm{~m}^{2}$ & $58.70(44.52-87.05)$ & $47.60(34.17-56.22)$ & $75.80(57.68-101.66)$ & $<0.001$ \\
\hline Hematuria, $n(\%)$ & $163(84.0)$ & $72(88.9)$ & $91(80.5)$ & 0.117 \\
\hline Hemoglobin, g/L & $121 \pm 25$ & $109 \pm 24$ & $130 \pm 21$ & $<0.001$ \\
\hline UACR, g/mmol & $1.79(0.64-3.99)$ & $3.60(2.16-5.93)$ & $0.86(0.26-2.20)$ & $<0.001$ \\
\hline 24-h proU, g & $2.40(1.04-5.42)$ & $5.11(2.56-8.69)$ & $1.25(0.63-2.67)$ & $<0.001$ \\
\hline Proteinuria $\geq 3.5 \mathrm{~g} / 24 \mathrm{~h}$ & 74 & $55(74 \%)$ & $19(26 \%)$ & $<0.001$ \\
\hline Proteinuria $<3.5 \mathrm{~g} / 24 \mathrm{~h}$ & 120 & $26(22 \%)$ & $94(78 \%)$ & $<0.001$ \\
\hline Albumin, g/L & $38 \pm 7$ & $35 \pm 6$ & $41 \pm 7$ & $<0.001$ \\
\hline $\mathrm{TC}, \mathrm{mmol} / \mathrm{L}$ & $5.30 \pm 1.74$ & $5.55 \pm 2.09$ & $5.13 \pm 1.34$ & 0.113 \\
\hline $\mathrm{TG}, \mathrm{mmol} / \mathrm{L}$ & $1.70(1.21-2.58)$ & $1.69(1.19-2.64)$ & $1.70(1.22-2.55)$ & 0.948 \\
\hline $\mathrm{HDL}-\mathrm{C}, \mathrm{mmol} / \mathrm{L}$ & $1.05 \pm 0.29$ & $1.11 \pm 0.31$ & $1.02 \pm 0.26$ & 0.123 \\
\hline LDL-C, mmol/L & $2.88(2.14-3.89)$ & $3.11(2.49-4.36)$ & $2.83(2.11-3.42)$ & 0.111 \\
\hline
\end{tabular}

DM, diabetes mellitus; OHA, oral hypoglycemic agents; RAAS, renin-angiotensin-aldosterone system; DR, diabetic retinopathy; SBP, systolic blood pressure; DBP, diastolic blood pressure; HbA1C, glycosylated hemoglobin; sCr, serum creatine; BUN, blood urea nitrogen; eGFR, estimated glomerular filtration rate; UACR, urinary microalbuminuria creatine ratio; $24-\mathrm{h}$ proU, $24-\mathrm{h}$ proteinuria; TC, total cholesterol; TG, triglycerides; HDL-C, high-density lipoprotein cholesterol; LDL-C, low-density lipoprotein cholesterol. $p<0.05$ was considered significant difference.

Whitney U tests, respectively. Kaplan-Meier curves were utilized to analyze cumulative renal survival, with comparisons between groups being made via the log-rank test. Independent predictors of renal outcomes were selected via a multivariate Cox proportional hazards regression model approach that was used to estimate hazard ratios (HRs) and 95\% confidence intervals (CIs). The results of this multivariate analysis were then used to guide the construction of a nomogram that was used to analyze the odds of reaching a defined renal endpoint within 1, 3, and 5 years. The predictive performance of this nomogram was analyzed using calibration curves, area under curve (AUC), and concordance index (C-index) values. In addition, a decision curve analysis (DCA) was performed in order to evaluate the net benefit of this predictive nomogram relative to other potential prognostic models. A 2-sided $p<0.05$ was the significance threshold.

Clinical-Pathological-Based Prognostic

Model in Diabetic Nephropathy

\section{Results}

\section{Patient Characteristics}

In total, among the 248 patients with renal biopsy-proven $\mathrm{DN}$ at the study hospital over the course of the study period, 194 were enrolled (shown in online suppl. Fig. 1). Patient baseline clinicopathological characteristics are shown in Tables 1 and 2. Patients were $70.6 \%$ male and had a median age of 54 years. Relative to patients that did not experience renal endpoint events during the study period, those that did exhibited more frequent insulin use, increased rates of DR, higher blood urea nitrogen, $\mathrm{sCr}$, systolic blood pressure, cystatin C, 24-h proteinuria, and UACR as well as lower eGFR, hemoglobin, and albumin levels (Table 1). 
Table 2. Pathological characteristics of patients with diabetic nephropathy

\begin{tabular}{|c|c|c|c|c|}
\hline \multirow[t]{2}{*}{ Variables } & \multirow{2}{*}{$\begin{array}{l}\text { Total patients } \\
(n=194)\end{array}$} & \multicolumn{2}{|c|}{ Renal endpoint events } & \multirow[t]{2}{*}{$p$ value } \\
\hline & & yes $(n=81)$ & no $(n=113)$ & \\
\hline \multicolumn{5}{|c|}{ Glomerular class, $n(\%)$} \\
\hline I & $12(6.2)$ & $0(0.0)$ & $12(10.6)$ & \multirow{5}{*}{$<0.001$} \\
\hline IIa & $33(17.0)$ & $2(2.5)$ & $31(27.4)$ & \\
\hline IIb & $38(19.6)$ & $7(8.6)$ & $31(27.4)$ & \\
\hline III & $101(52.1)$ & $65(80.2)$ & $36(31.9)$ & \\
\hline IV & $10(5.2)$ & $7(8.6)$ & $3(2.7)$ & \\
\hline \multicolumn{5}{|c|}{ IFTA, $n(\%)$} \\
\hline 0 & $4(2.1)$ & $0(0.0)$ & $4(3.5)$ & \multirow{4}{*}{$<0.001$} \\
\hline 1 & $67(34.5)$ & $5(6.2)$ & $62(54.9)$ & \\
\hline 2 & $81(41.8)$ & $40(49.4)$ & $41(36.3)$ & \\
\hline 3 & $42(21.6)$ & $36(44.4)$ & $6(5.3)$ & \\
\hline \multicolumn{5}{|c|}{ Interstitial inflammation, $n(\%)$} \\
\hline 0 & $7(3.6)$ & $0(0.0)$ & $7(6.2)$ & \multirow{3}{*}{$<0.001$} \\
\hline 1 & $133(68.6)$ & $35(43.2)$ & $98(66.7)$ & \\
\hline 2 & $54(27.8)$ & $46(56.8)$ & $8(7.1)$ & \\
\hline \multicolumn{5}{|c|}{ Arteriolar hyalinosis, $n(\%)$} \\
\hline 0 & $19(9.8)$ & $1(1.2)$ & $18(15.9)$ & \multirow{3}{*}{$<0.001$} \\
\hline 1 & $93(47.9)$ & $35(43.2)$ & $58(51.3)$ & \\
\hline 2 & $82(42.3)$ & $45(55.6)$ & $37(32.7)$ & \\
\hline \multicolumn{5}{|c|}{ Arteriosclerosis, $n(\%)$} \\
\hline 0 & $58(29.9)$ & $14(17.3)$ & $44(38.9)$ & \multirow{3}{*}{0.001} \\
\hline 1 & $86(44.3)$ & $37(45.7)$ & $49(43.4)$ & \\
\hline 2 & $50(25.8)$ & $30(37.0)$ & $20(17.7)$ & \\
\hline
\end{tabular}

IFTA, interstitial fibrosis and tubular atrophy. $p<0.05$ was considered significant difference.

Pathological findings in analyzed patients are compiled in Table 2. Patients who experienced renal endpoint events exhibited more severe glomerular injury, more severe IFTA, increased levels of interstitial inflammation, and increased incidence of arteriolar hyalinosis and arteriosclerosis.

\section{Identification of Predictors of Renal Survival}

A total of $81 \mathrm{DN}$ patients suffered from renal endpoint events during the follow-up (median 30 months). In total, univariate Cox analyses identified 14 baseline clinicopathologic parameters that were associated with renal endpoint events ( $p<0.05$; online suppl. Table 1$)$, whereas only 4 of these parameters were identified as significant independent predictors of renal outcomes in a multivariate Cox analysis, including CKD stages (HR: 1.445; 95\% CI: $1.157-1.806 ; p=0.001$ ), 24 -h proteinuria (HR: 1.114 ; 95\% CI: $1.051-1.180 ; p<0.001$ ), glomerular class (HR: 2.163; 95\% CI: 1.456-3.212; $p<0.001$ ), and IFTA scores (HR: 1.602; 95\% CI: 1.105-2.322; $p=0.013$ ) (Table 3). As shown in Figure 1a-d, DN patients exhibiting nephroticrange proteinuria ( $\geq 3.5 \mathrm{~g}$ /day), later-stage $\mathrm{CKD}$ (CKD
3-4), more severe glomerular lesions (glomerular classification III-IV), or increased IFTA scores (score 2-3) experienced significantly worse renal outcomes.

\section{Prognostic Nomogram Development}

We next developed a nomogram incorporating these 4 independent predictors of DN patient renal outcomes in order to provide an easy-to-use tool for patient risk stratification (shown in Fig. 2). In this nomogram, points were assigned by following a line upward from each axis to the "points" axis on the top of the nomogram. These points were added together for a given patient to yield a total score which could then be used to gauge their risk of reaching a renal endpoint within a given period of time based on the scale at the bottom of the nomogram. For example, a DN patient with glomerular class II lesions (50 points), an IFTA score of 2 (30 points), a proteinuria level of $2 \mathrm{~g} / 24 \mathrm{~h}$ (7 points), and a diagnosis of CKD stage 2 (12 points) would have a total of 99 points, which corresponds to an estimated 1-, 3-, and 5-year renal survival likelihood of $100 \%, 82 \%$, and $74 \%$, respectively.
Zhou/Wang/Shen/Li/Jiao/Li/Jia/He/ Zhang/Wang/Fan 


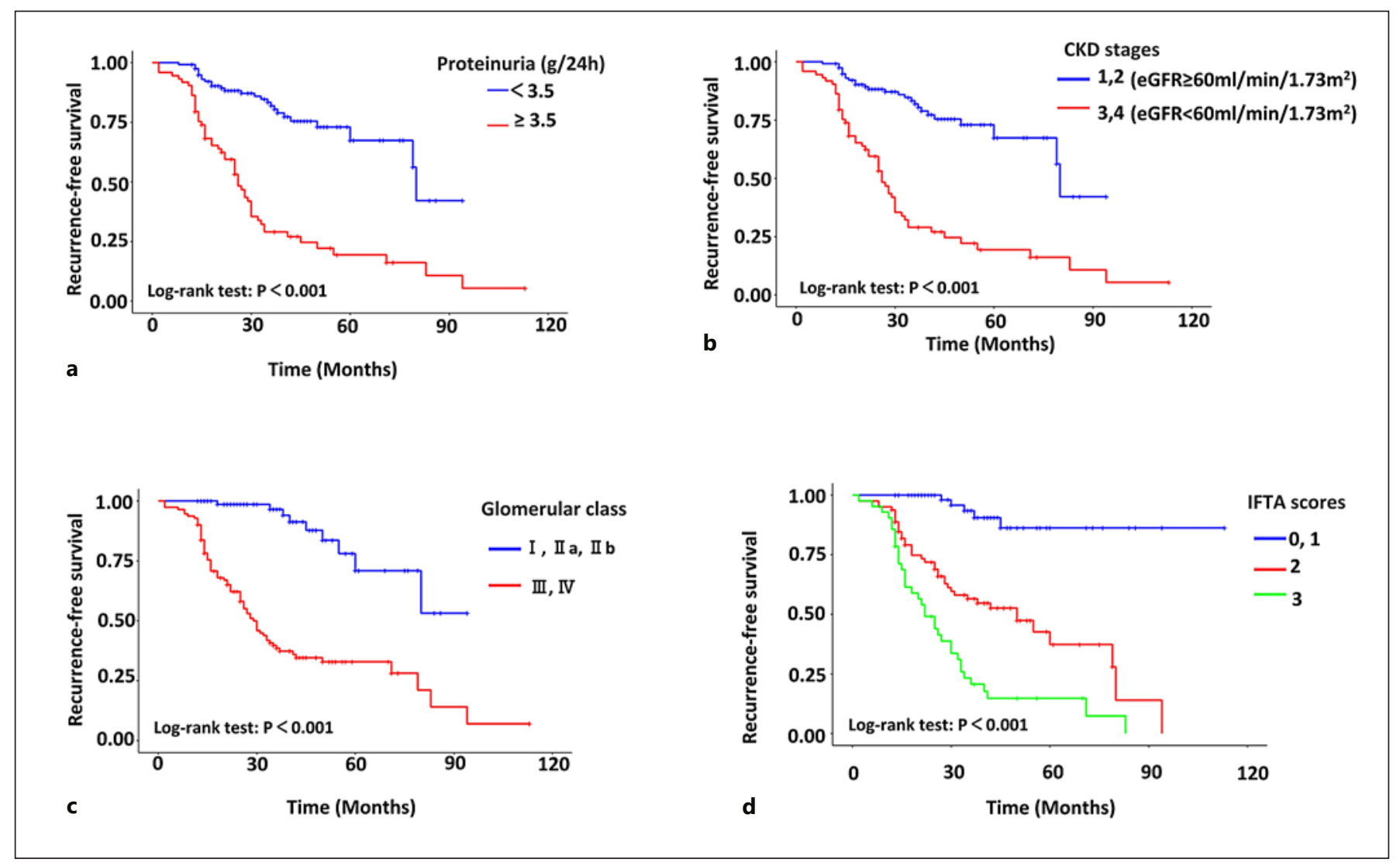

Fig. 1. Patient survival as a function of different clinicopathologic characteristics. Proteinuria (a), CKD stages (b), glomerular class (c), and IFTA scores (d). CKD, chronic kidney disease; CKD stages 1, 2: eGFR >60 mL/ $\mathrm{min} / 1.73 \mathrm{~m}^{2}$; CKD stages 3, 4: $30<\mathrm{eGFR} \leq 60 \mathrm{~mL} / \mathrm{min} / 1.73 \mathrm{~m}^{2}$; IFTA, interstitial fibrosis and tubular atrophy.

\section{Nomogram Calibration and Assessment}

This nomogram yielded a C-index value of 0.845 (95\% CI: $0.826-0.864)$ upon internal validation, consistent with good discriminatory ability, and the 3-year and 5-year AUC and 95\% CI were 0.933 (95\% CI: 0.898-0.968) and 0.923 (95\% CI: 0.886-0.960). Calibration curves also revealed good consistency between actual and predicted renal survival over 3- and 5-year periods (shown in Fig. 3; online suppl. Fig. 2).

\section{Assessment of Model Performance}

To evaluate the clinical utility and performance of our model, we compared the predicted probabilities of renal endpoint events predicted using our model to those predicted based upon CKD stages or 24-h proteinuria only. This DCA approach revealed that when the probability threshold for a patient was from 10 to 95\% for 3-year renal endpoints and from 20 to $98 \%$ for 5 -year renal endpoints, our clinical nomogram offered
Table 3. Multivariate Cox regression analysis for the prediction of renal survival

\begin{tabular}{llllr}
\hline Variables & $\beta$-estimate & HR & 95\% CI & $p$ value \\
\hline CKD stages & 0.368 & 1.445 & $1.157-1.806$ & 0.001 \\
24-h proU & 0.108 & 1.114 & $1.051-1.180$ & $<0.001$ \\
Glomerular class & 0.771 & 2.163 & $1.456-3.212$ & $<0.001$ \\
IFTA scores & 0.471 & 1.602 & $1.105-2.322$ & 0.013 \\
\hline
\end{tabular}

CKD, chronic kidney disease; 24-h proU, 24-h proteinuria; IFTA, interstitial fibrosis and tubular atrophy. $p<0.05$ was considered significant difference.

a net benefit relative to a "treat all" or "treat none" approach (shown in Fig. 4a, b). Importantly, our model also offered greater net benefit and exhibited superior clinical performance relative to those models based solely upon CKD stages or 24 -h proteinuria, indicat- 


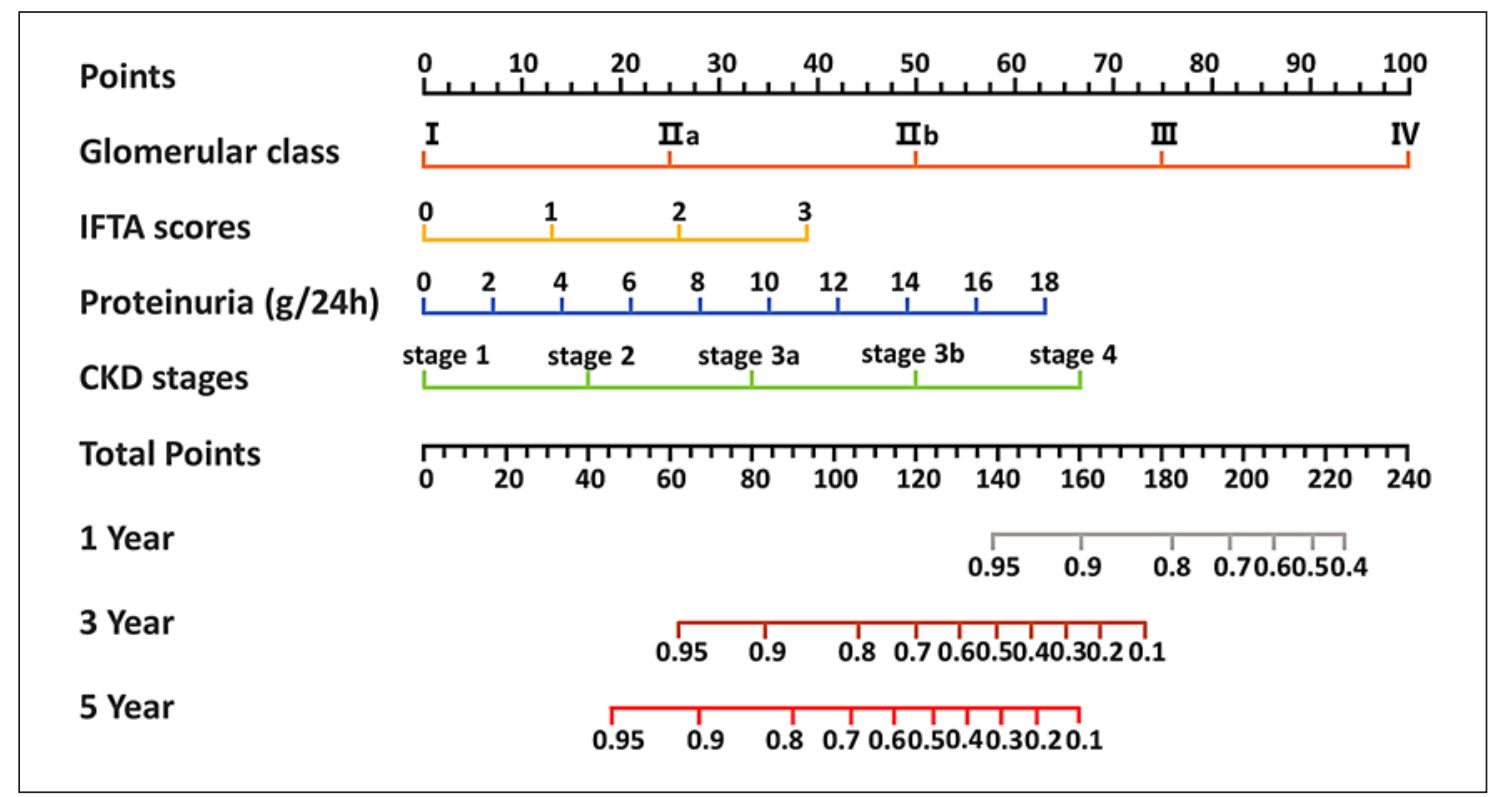

Fig. 2. Prediction of the individual odds of renal survival in diabetic nephropathy patients using a prognostic nomogram. This predictive nomogram can be used to predict the odds of 1-, 3-, and 5-year renal survival based on patient-specific values for the included variables. Point values are determined by following a straight line upward from the appropriate value for a given variable to the "points" line. Total point values are calculated and can then be used to estimate survival values by following a straight line down to the corresponding survival probability axes. IFTA, interstitial fibrosis and tubular atrophy; CKD, chronic kidney disease.

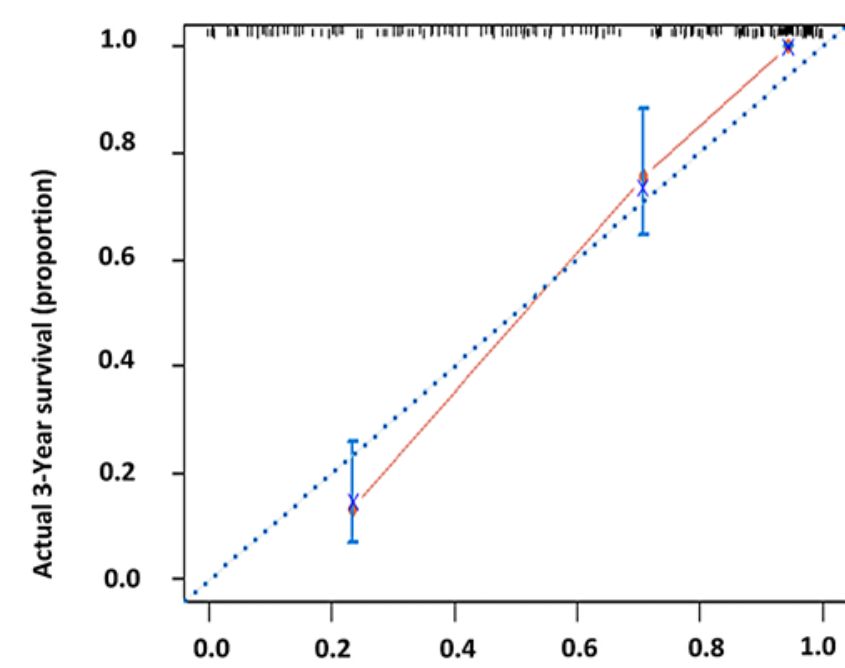

a

Nomogram-Predicted Probability of 3-Year

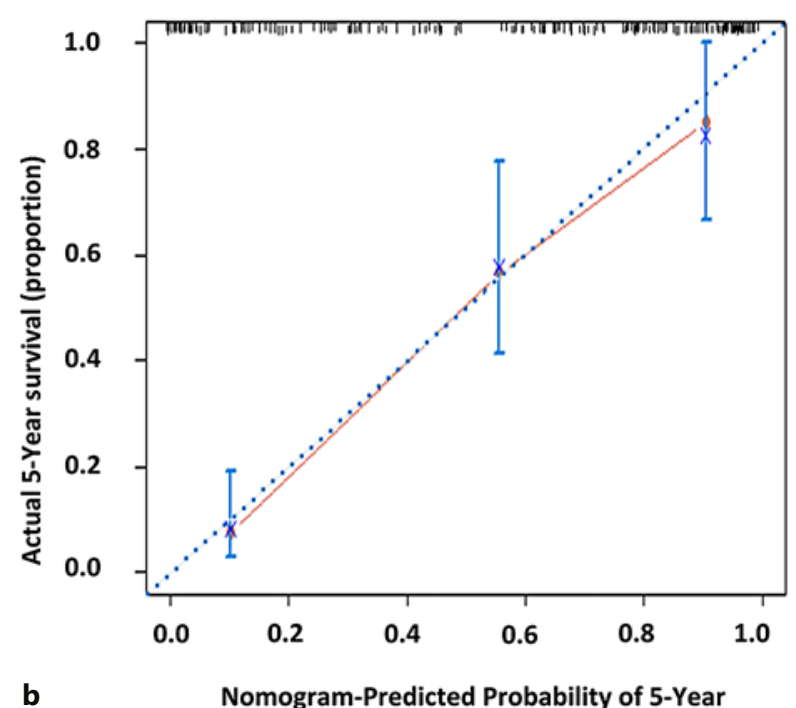

b

Fig. 3. Calibration curves for a nomogram predicting renal survival in diabetic nephropathy patients. Calibration curves show 3-year (a) and 5-year (b) outcomes. Odds of renal survival based on nomogram predictions are shown on the $x$-axis, with actual renal survival on the $y$-axis. 


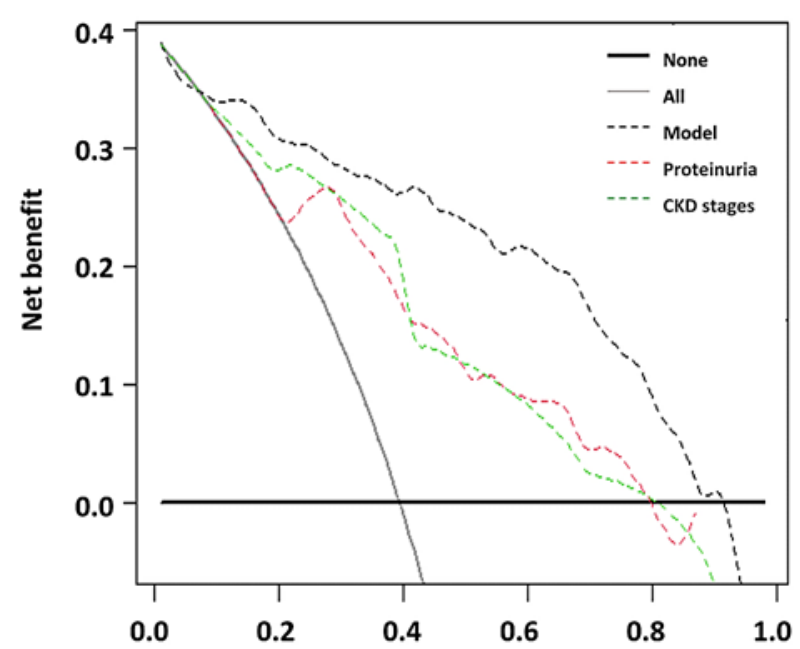

a

Threshold probability of 3-Year

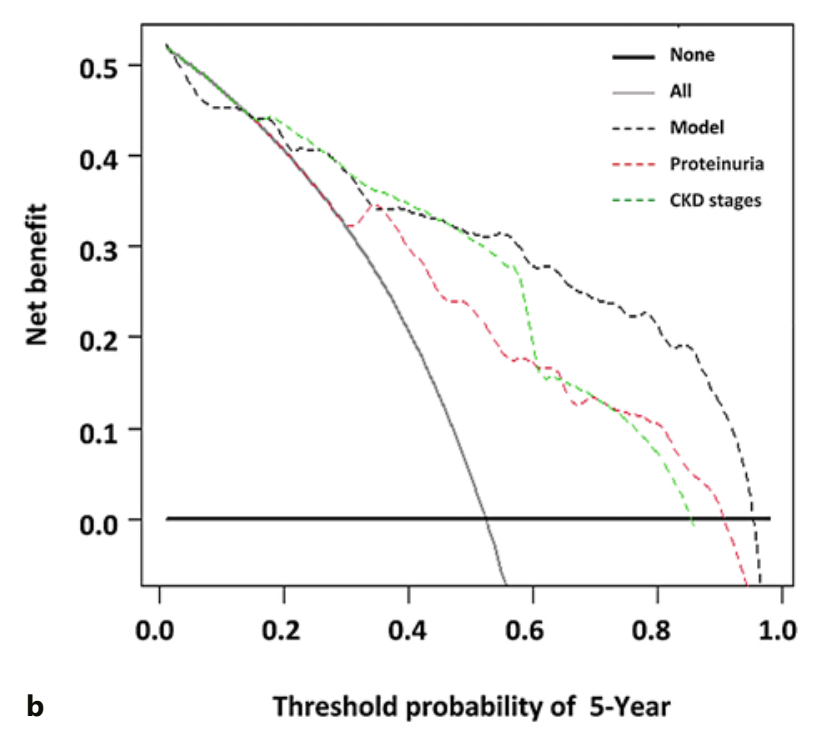

Fig. 4. Decision curve analyses comparing the relative utility of our prognostic nomogram, CKD stages, and proteinuria status in DN patients. Three-year DCA (a); 5-year DCA (b). Net benefit curves are shown for nomogram, proteinuria, and CKD stages. Solid black lines indicate the net benefit when all DN patients are considered as not exhibiting a renal outcome, whereas solid gray lines correspond to the net benefit when all DN patients are considered as exhibiting a renal outcome. The optimal model is that which yields the greatest net benefit at a given threshold. CKD, chronic kidney disease; DN, diabetic nephropathy; DCA, decision curve analysis.

ing that our nomogram is a reliable clinical tool that can be used to predict DN patient renal endpoint events.

\section{Discussion}

In the current study, we assessed the association between clinicopathological parameters and renal outcomes in patients with biopsy-confirmed DN. We found that renal survival was independently associated with CKD stages, 24 -h proteinuria, glomerular class, and IFTA scores in these patients. A prognostic nomogram incorporating these 4 variables was then developed and used to accurately and quantitatively analyze the risk of a given patient experiencing an adverse renal outcome. This model was reliable as a tool for prediction of renal failure, performing better than models based solely upon clinical parameters.

Multiple clinical factors have been associated with an increased chance of renal failure in DN patients to date, with the 2012 KDIGO guidelines having identified eGFR and proteinuria as the 2 primary prognostic indicators of diabetic ESRD risk [17]. In the present study, we graded patient baseline $24-\mathrm{h}$ proteinuria and eGFR, treating them as categorical variables in order to enable them to be easily incorporated into our predictive nomogram. We found both of these variables are independent predictors of patient renal survival, consistent with prior results [18]. While other factors including hematuria [19] and the presence of DR [20] have been identified as clinical biomarkers of DN patient renal prognosis in prior studies, these results remain controversial. In their analysis of 111 patients, Li et al. [21] identified hematuria as an independent predictor of DN progression, whereas Shimizu et al. [11] detected no such relationship between hematuria and renal event risk in their study of 260 individuals with biopsy-confirmed DN. DR has also been shown to be a valuable predictor of renal outcomes in an analysis of 232 patients with biopsy-confirmed DN [22]. However, we did not identify significant association between hematuria and renal outcomes. While these variables were associated with one another in univariate analyses, this relationship was no longer evident following multivariate analysis. 
Renal structural findings are also closely linked to DN progression. Glomerular lesions, in particular, are the most apparent pathological findings associated with $\mathrm{DN}$, and they have previously been shown to be reliable predictors of renal events $[23,24]$. These results, however, are not universal, with 1 small study of 69 patients with biopsy-confirmed DN having found these glomerular lesions to be unrelated to renal outcomes [25]. Herein, we found that patients with class III and IV lesions exhibited significantly worse renal survival relative to those with class I and II lesions, and we confirmed that such lesion severity was an independent predictor of renal outcomes. We also confirmed that IFTA was predictive of these renal outcomes in patients with biopsy-confirmed DN, consistent with the results of a previous large study of 396 such patients [15]. However, IFTA was found to only be of predictive value in univariate analyses and to be unrelated to cortical area or the rate of eGFR decline in prior analyses [26,27]. These inconsistencies, however, may be related to the small sample size of these studies or to differences in assessment methodology.

Given that clinical or pathological changes may be insufficient to fully evaluate the progression and prognosis of $\mathrm{DN}$ when evaluated in isolation, predictive nomograms represent an ideal tool for evaluating the relationship between clinicopathologic features and time to ESRD in those with biopsy-confirmed DN $[18,28]$. We incorporated 4 different variables into our nomogram (24-h proteinuria, CKD stages, glomerular lesions, and IFTA scores), all of which can be easily measured in a clinical setting. Calibration curves confirmed the accuracy of our nomogram when used to analyze both the primary patient cohort and internal validation cohorts at 1-, 3-, and 5 -year endpoints. A DCA approach further confirmed that this clinicopathological nomogram was superior to other models based solely upon clinical indicators as a tool for predicting renal survival in DN patients.

There are multiple strengths to this model. For one, this nomogram is easy to use and relies upon both clinical and pathologic indicators, offering predictive value that has been validated for multiple stages of DN. In addition, a DCA approach revealed that our nomogram offered significantly better performance relative to other models based solely on traditional clinical markers.

There are multiple limitations to the present study. For one, this was a single-center analysis with a small sample size that was thus susceptible to selection bias. Further multicenter analyses will therefore be essential to validate the clinical utility of this model in independent external cohorts. Second, the pathologic changes evaluated in this study were based upon pathological classifications of DN, while other pathological signs that are strongly predictive of ESRD, including exudative lesions and mesangial matrix volume fraction $[29,30]$, have not been evaluated here. Incorporating these pathological variables into future studies may improve the prognostic utility of our nomogram. Last, as our study was restricted to an Asian patient population with type 2 diabetes, these findings may not be generalizable, and our predictive nomogram is based on data from biopsy-proven diabetic nephropathy patients, which may not represent the progression of every DN patient as currently there are still many patients who were clinically diagnosed as DN.

In summary, we developed a straightforward and effective nomogram that incorporated both clinical and pathological variables and that was capable of predicting renal survival in patients with biopsy-confirmed $\mathrm{DN}$. We believe that this model will guide future research regarding the progression of this disease, aiding clinicians to provide patients with appropriate treatment options.

\section{Statement of Ethics}

The Ethics Committee of the Shanghai Jiao Tong University Affiliated Sixth People's Hospital approved this study (2016-41), which was consistent with the Declaration of Helsinki. Written informed consent was obtained from all patients.

\section{Conflict of Interest Statement}

The authors declare that there are no conflicts of interest that could be perceived as prejudicing the impartiality of the research reported.

\section{Funding Sources}

This study was supported by the National Nature Science Foundation of China (81670657, 81870504, and 81870468), the Shanghai Jiao Tong University Gaofeng Talent Training Plan, and a clinical project (20192833), the Interdisciplinary Program of Shanghai Jiao Tong University (YG2017MS10), Open Project of Shanghai Key Laboratory of Sleep Disordered Breathing (SHKSDB-KF-19-04), Science and Technology Commission of Shanghai Municipality (14DZ2260200, the project of Shanghai Key Laboratory of Kidney and Blood Purification), Three-year Project of Shanghai TCM Development (ZT[2018-2020]-FWTX-2003), and Star Program of Shanghai Jiao Tong University (20190102). 


\section{Author Contributions}

Ying Fan and Niansong Wang designed the research project. Yiyun Wang, Ting Zhou, Qiming Zhang, Xiaomei Li, Qiong Jiao, $\mathrm{Ze} \mathrm{Li}$, Junjie Jia, and Li He performed the study, collected the clinical data, and worked on the follow-up of patients. Ting Zhou, Yiyun Wang, and Li Shen analyzed the data. Ting Zhou and Yiyun Wang drafted the manuscript. Ying Fan and Niansong Wang revised and approved the final version of the manuscript.

\section{Data Availability Statement}

All data generated or analyzed during this study are included in this article and its online suppl. Files. Further enquiries can be directed to the corresponding author.

\section{References}

1 Alicic RZ, Rooney MT, Tuttle KR. Diabetic kidney disease: challenges, progress, and possibilities. Clin J Am Soc Nephrol. 2017 Dec; 12(12):2032-45.

2 Saeedi P, Petersohn I, Salpea P, Malanda B, Karuranga S, Unwin N, et al. Global and regional diabetes prevalence estimates for 2019 and projections for 2030 and 2045: results from the International Diabetes Federation Diabetes Atlas, 9(th) edition. Diabetes Res Clin Pract. 2019 Nov;157:107843.

3 Giugliano D, Maiorino MI, Bellastella G, Esposito K. Comment on American Diabetes Association. approaches to glycemic treatment. Sec. 7. In standards of medical care in diabetes-2016. Diabetes care 2016;39(Suppl. 1):S52-S59. Diabetes Care. 2016 Jun;39(6): e86-7.

4 Berhane AM, Weil EJ, Knowler WC, Nelson RG, Hanson RL. Albuminuria and estimated glomerular filtration rate as predictors of diabetic end-stage renal disease and death. Clin J Am Soc Nephrol. 2011 Oct;6(10):2444-51.

5 Andrassy KM. Comments on "KDIGO 2012 clinical practice guideline for the evaluation and management of chronic kidney disease." Kidney Int. 2013 Sep;84(3):622-3.

6 Ninomiya T, Perkovic V, de Galan BE, Zoungas S, Pillai A, Jardine M, et al. Albuminuria and kidney function independently predict cardiovascular and renal outcomes in diabetes. J Am Soc Nephrol. 2009 Aug;20(8):181321.

7 Pichaiwong W, Homsuwan W, Leelahavanichkul A. The prevalence of normoalbuminuria and renal impairment in type 2 dia-

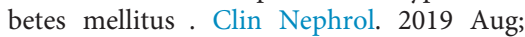
92(2):73-80.

8 Dwyer JP, Parving HH, Hunsicker LG, Ravid M, Remuzzi G, Lewis JB. Renal dysfunction in the presence of normoalbuminuria in type 2 diabetes: results from the DEMAND Study. Cardiorenal Med. 2012 Feb;2(1):1-10.

9 Caramori ML, Fioretto P, Mauer M. Low glomerular filtration rate in normoalbuminuric type 1 diabetic patients: an indicator of more advanced glomerular lesions. Diabetes. 2003 Apr;52(4):1036-40.

10 Macisaac RJ, Jerums G. Diabetic kidney disease with and without albuminuria. Curr Opin Nephrol Hypertens. 2011 May;20(3): 246-57.
11 Shimizu M, Furuichi K, Toyama T, Kitajima S, Hara A, Kitagawa K, et al. Long-term outcomes of Japanese type 2 diabetic patients with biopsy-proven diabetic nephropathy. Diabetes Care. 2013 Nov;36(11):3655-62.

12 Comai G, Malvi D, Angeletti A, Vasuri F, Valente S, Ambrosi F, et al. Histological evidence of diabetic kidney disease precede clinical diagnosis. Am J Nephrol. 2019;50(1):29-36.

13 Klessens CQ, Woutman TD, Veraar KA, Zandbergen M, Valk EJ, Rotmans JI, et al. An autopsy study suggests that diabetic nephropathy is underdiagnosed. Kidney Int. 2016 Jul; 90(1):149-56.

14 Tervaert TW, Mooyaart AL, Amann K, Cohen $\mathrm{AH}$, Cook HT, Drachenberg CB, et al. Pathologic classification of diabetic nephropathy. J Am Soc Nephrol. 2010 Apr;21(4):55663.

15 An Y, Xu F, Le W, Ge Y, Zhou M, Chen H, et al. Renal histologic changes and the outcome in patients with diabetic nephropathy. Nephrol Dial Transplant. 2015 Feb;30(2):257-66.

16 Tan GS, Gan A, Sabanayagam C, Tham YC, Neelam K, Mitchell P, et al. Ethnic differences in the prevalence and risk factors of diabetic retinopathy: the Singapore Epidemiology of Eye Diseases Study. Ophthalmology. 2018 Apr;125(4):529-36.

17 Inker LA, Astor BC, Fox CH, Isakova T, Lash JP, Peralta CA, et al. KDOQI US commentary on the 2012 KDIGO clinical practice guideline for the evaluation and management of CKD. Am J Kidney Dis. 2014 May;63(5):71335.

18 Vernerey D, Huguet F, Vienot A, Goldstein D, Paget-Bailly S, Van Laethem JL, et al. Prognostic nomogram and score to predict overall survival in locally advanced untreated pancreatic cancer (PROLAP). Br J Cancer. 2016 Jul 26;115(3):281-9.

19 Lin HY, Niu SW, Kuo IC, Lim LM, Hwang DY, Lee JJ, et al. Hematuria and renal outcomes in patients with diabetic chronic kidneydisease. Am J Med Sci. 2018 Sep;356(3): 268-76.

20 Hung CC, Lin HY, Hwang DY, Kuo IC, Chiu YW, Lim LM, et al. Diabetic retinopathy and clinical parameters favoring the presence of diabetic nephropathy could predict renal outcome in patients with diabetic kidney disease. Sci Rep. 2017 Apr 21;7(1):1236.
21 Zhuo L, Zhang N, Zou G, Chen D, Li W. Clinical characteristics and outcomes of biopsyproven diabetic nephropathy. Front Med. 2017 Sep;11(3):386-92.

22 Yamanouchi M, Mori M, Hoshino J, Kinowaki K, Fujii T, Ohashi K, et al. Retinopathy progression and the risk of end-stage kidney disease: results from a longitudinal Japanese cohort of 232 patients with type 2 diabetes and biopsy-proven diabetic kidney disease. BMJ Open Diabetes Res Care. 2019;7(1):e000726.

23 Oh SW, Kim S, Na KY, Chae DW, Kim S, Jin DC, et al. Clinical implications of pathologic diagnosis and classification for diabetic nephropathy. Diabetes Res Clin Pract. 2012 Sep; 97(3):418-24.

24 Mise K, Hoshino J, Ubara Y, Sumida K, Hiramatsu R, Hasegawa E, et al. Renal prognosis a long time after renal biopsy on patients with diabetic nephropathy. Nephrol Dial Transplant. 2014 Jan;29(1):109-18.

25 Okada T, Nagao T, Matsumoto H, Nagaoka Y, Wada T, Nakao T. Histological predictors for renal prognosis in diabetic nephropathy in diabetes mellitus type 2 patients with overt proteinuria. Nephrology. 2012 Jan;17(1):6875.

26 Ruggenenti P, Gambara V, Perna A, Bertani T, Remuzzi G. The nephropathy of non-insulin-dependent diabetes: predictors of outcome relative to diverse patterns of renal injury. J Am Soc Nephrol. 1998 Dec;9(12): 2336-43.

27 Christensen PK, Larsen S, Horn T, Olsen S, Parving HH. Renal function and structure in albuminuric type 2 diabetic patients without retinopathy. Nephrol Dial Transplant. 2001 Dec;16(12):2337-47.

28 Balachandran VP, Gonen M, Smith JJ, DeMatteo RP. Nomograms in oncology: more than meets the eye. Lancet Oncol. 2015 Apr; 16(4):e173-80.

29 Furuichi K, Yuzawa Y, Shimizu M, Hara A, Toyama T, Kitamura H, et al. Nationwide multicentre kidney biopsy study of Japanese patients with type 2 diabetes. Nephrol Dial Transplant. 2018 Jan 1;33(1):138-48.

30 Perrin NE, Torbjörnsdotter TB, Jaremko GA, Berg UB. The course of diabetic glomerulopathy in patients with type I diabetes: a 6-year follow-up with serial biopsies. Kidney Int. 2006 Feb;69(4):699-705. 